Original paper

\title{
Prevalence and prognostic value of cirrhotic cardiomyopathy as defined according to the proposed new classification
}

\author{
Maurizio Cesari', Anna Chiara Frigo², Salvatore Piano' ${ }^{1}$ Paolo Angeli \\ 'Unit of Internal Medicine and Hepatology, Department of Medicine, University of Padua, Italy \\ 2Department of Cardiac, Thoracic and Vascular Sciences, University of Padua, Padua, Italy
}

\begin{abstract}
Aim of the study: Prevalence and prognostic relevance of cirrhotic cardiomyopathy (CCM), as defined according to the new core criteria proposed in 2019, are still unknown. We investigated this relevant issue in a large cohort of cirrhotic patients.

Material and methods: We retrospectively interrogated a data set of 162 collected cirrhotic patients followed up for at least 6 years, who underwent standard Doppler echocardiography and were compared with 46 healthy subjects. Left ventricular (LV) geometry, systo-diastolic function, global longitudinal strain and the main hemodynamic parameters were assessed according to current guidelines. Systolic dysfunction was diagnosed if LV ejection fraction (LVEF) $\leqslant 50 \%$ and/or global longitudinal strain $(G L S)<18 \%$ or $>22 \%$.

Results: Adequate echocardiographic images permitting speckle tracking analysis were available in 83 patients. No patient presented LVEF $\leqslant 50 \%$, GLS $<18 \%$ or $>22 \%$ was evident in $25 \%$, advanced diastolic dysfunction was evident in $10 \%$. Overall the prevalence of CCM was $29 \%$. Patients with and without CCM presented similar clinical, biochemical, hemodynamic and echocardiographic features at baseline and similar incidence of death or type 1 hepatorenal syndrome at follow-up.

Conclusions: According to the new criteria CCM is detected in $29 \%$, mainly due to altered GLS at rest, but without prognostic relevance and therefore useless for the clinical management of cirrhotic patients. We propose to modify the criteria by removing the LVEF assessment and adding also a stress test assessing the cardiac contractile reserve to distinguish patients with a blunted cardiac response, which could present a worst prognosis.

Key words: cirrhosis, echocardiography, systolic function, diastolic dysfunction, cirrhotic cardiomyopathy.
\end{abstract}

Address for correspondence:

Dr. Maurizio Cesari, Unit of Internal Medicine and Hepatology, Department of Medicine, Policlinico Universitario,

Clinica Medica 5, Via Giustiniani 2, 35125 Padova, Italy, e-mail: maurizio.cesari@unipd.it

\section{Introduction}

First defined in 2005 at the Montreal World Congress of Gastroenterology, cirrhotic cardiomyopathy (CCM) is a condition of subclinical cardiac dysfunction characterized by impaired myocardial contractility, left ventricular (LV) hypertrophy, diastolic dysfunction (DD), impaired chronotropic function and electrophysiological abnormalities in the absence of other known causes of heart disease [1]. The preliminary cri- teria which were considered for the diagnosis of CCM are reported in Table 1 . This condition has been reported to predict the development of hepatorenal syndrome (HRS) [2] and poor outcome in patients with advanced cirrhosis and in patients undergoing orthotopic liver transplantation (OLT) [3]. CCM is actually considered a condition of latent heart failure which manifests only under stress, resulting in a blunted increase in cardiac index (CI) and cardiac output (CO) during exercise or pharmacologic stimuli $[1,4]$, but which is barely iden- 
Table 1. Criteria for cirrhotic cardiomyopathy (CCM) as proposed at the World Congress of Gastroenterology in 2005

\begin{tabular}{lcc}
\hline Systolic dysfunction & Diastolic dysfunction & Supporting criteria \\
\hline Any of the following & Any of the following & Electrophysiological abnormalities \\
\hline & & Abnormal chronotropic response \\
\hline Blunted contractile response on stress testing & Deceleration time of the E wave $>200 \mathrm{~ms}$ & Electromechanical uncoupling \\
\hline Left ventricular ejection fraction $<55 \%$ & Isovolumetric relaxation time $>80 \mathrm{~ms}$ & Prolonged QTc interval \\
\hline & E/A $<1$ & Enlarged left atrium \\
\hline & & Increased myocardial mass \\
\hline
\end{tabular}

$B N P$ - brain natriuretic peptide, E/A - early and late diastolic velocity ratio at transmitral Doppler

tified at rest mainly because it is confused with other symptoms of advanced liver cirrhosis, such as exercise intolerance, fatigue and dyspnoea, and therefore its prevalence is still unclear. In the last decade 2-dimensional speckle tracking echocardiography (2DSTE) emerged as robust ultrasonic technique also validated by magnetic resonance imaging [5] allowing evaluation of the longitudinal function (deformation) of the myocardium through strain and strain rate analysis and providing information on subclinical impairment of longitudinal ventricular function [6]. The latter always precedes the depression of LV ejection fraction (LVEF) and may be a guide to the presence of fibrosis. Global longitudinal strain (GLS), a parameter derived from 2DSTE, has been reported to add incremental prognostic value, in addition to established prediction models, in the general population [6].

Hence, these advances in cardiovascular imaging, including the assessment of LV strain (longitudinal, radial and transverse), have provided new criteria to identify a subclinical cardiac dysfunction, rendering the 2005 CCM criteria obsolete. Therefore, in 2019 a position paper of a multi-disciplinary international group (Cirrhotic Cardiomyopathy Consortium) proposed new criteria to define CCM (Table 2), including advanced DD and systolic dysfunction defined as $\mathrm{LVEF} \leq 50 \%$ or absolute GLS $<18 \%$ or $>22 \%$ [7].

At present, no information exists as regards the prevalence of CCM according to these new criteria and, more importantly, its prognostic value in cirrhotic patients. Therefore, the aim of our study is to assess the real prevalence of CCM, as defined according to the above criteria, and its prognostic relevance after long follow-up.

\section{Material and methods}

\section{Patient selection}

We retrospectively interrogated a large data set of 162 consecutive cirrhotic patients collected from 2009 to 2012, who underwent a detailed haemodynamic evaluation and standard trans-thoracic Doppler echocardiography in our clinic. Exclusion criteria were arterial hypertension, history of cardiovascular disease, diabetes, obesity, and heart valve disease. Coronary heart disease was excluded in all participants on the

Table 2. Criteria for cirrhotic cardiomyopathy (CCM) as proposed by the Cirrhotic Cardiomyopathy Consortium (2019)

\begin{tabular}{|c|c|c|}
\hline Systolic dysfunction & Advanced diastolic dysfunction & Areas for future research which require further validation \\
\hline \multirow[t]{2}{*}{ Any of the following } & $\geqslant 3$ of the following & Abnormal chronotropic or inotropic response \\
\hline & & Electrocardiographic changes \\
\hline Left ventricular ejection fraction $\leqslant 50 \%$ & Septal e' velocity $<7 \mathrm{~cm} / \mathrm{s}$ & Electromechanical uncoupling \\
\hline \multirow{4}{*}{$\begin{array}{l}\text { Absolute global longitudinal strain } \\
<18 \% \text { or }>22 \%\end{array}$} & E/e' ratio $>15$ & Myocardial mass change \\
\hline & Left atrial volume index $>34 \mathrm{ml} / \mathrm{m}^{2}$ & Serum biomarkers \\
\hline & Tricuspid regurgitation velocity $>2.8 \mathrm{~m} / \mathrm{s}$ & Chamber enlargement \\
\hline & & Cardiac magnetic resonance imaging \\
\hline
\end{tabular}


basis of symptoms, negative family history, a normal standard 12-lead electrocardiogram, and normal wall motion on the two-dimensional echocardiographic examination.

If taken, $\beta$-blockers were stopped 48 hours before echocardiography. The diagnosis of liver cirrhosis was based on clinical, biochemical, imaging and endoscopic findings in all patients. The presence of ascites was detected clinically and confirmed by an abdomen ultrasound examination. The diagnosis of refractory ascites was based on the criteria of the International Club of Ascites [8]. In patients with refractory ascites the haemodynamic evaluation was performed soon after the therapeutic paracentesis.

As controls, we selected 46 healthy subjects, matched for age, who voluntarily participated in this study. All patients furnished written consent to the study, which was approved by the Ethics Committee of the University of Padua, and followed the Helsinki Declaration principles.

\section{Analysis of echocardiographic parameters}

All echocardiographic examinations were performed by an expert sonographer (M.C.) using a General
Electrics Vivid 7 ultrasound machine with a $2.5 \mathrm{MHz}$ transducer. Measurements were performed according to the American Society of Echocardiography/European Association of Echocardiography guidelines [9]. Left ventricular EF and fractional shortening (FS) were measured in biplane two-dimensional mode using Simpson's method [9]. Midwall fractional shortening (MWFS) was also calculated to assess underlying systolic dysfunction in the setting of concentric hypertrophy [10]. The LV mass (LVM) was estimated using the formula of Devereux et al. [9] and normalized both by body surface area (BSA) and by height in metres to the power of 2.7. Criteria for LV hypertrophy (LVH) were LVM/height $\geq 50 \mathrm{~g} / \mathrm{m}^{2.7}$ for men and $\geq 47 \mathrm{~g} / \mathrm{m}^{2.7}$ for women according to the current European Society of Cardiology/European Society of Hypertension (ESC/ ESH) guidelines.

The GLS was assessed according to the European Association of Cardiovascular Imaging (EACVI) and the American Society of Echocardiography (ASE) guidelines [11]. Figure 1 shows the global longitudinal strain polar map image of a cirrhotic patient with preserved systolic function and normal GLS.

Pulsed Doppler recordings at the level of the mitral valve tips were obtained from apical 4 -chamber scans
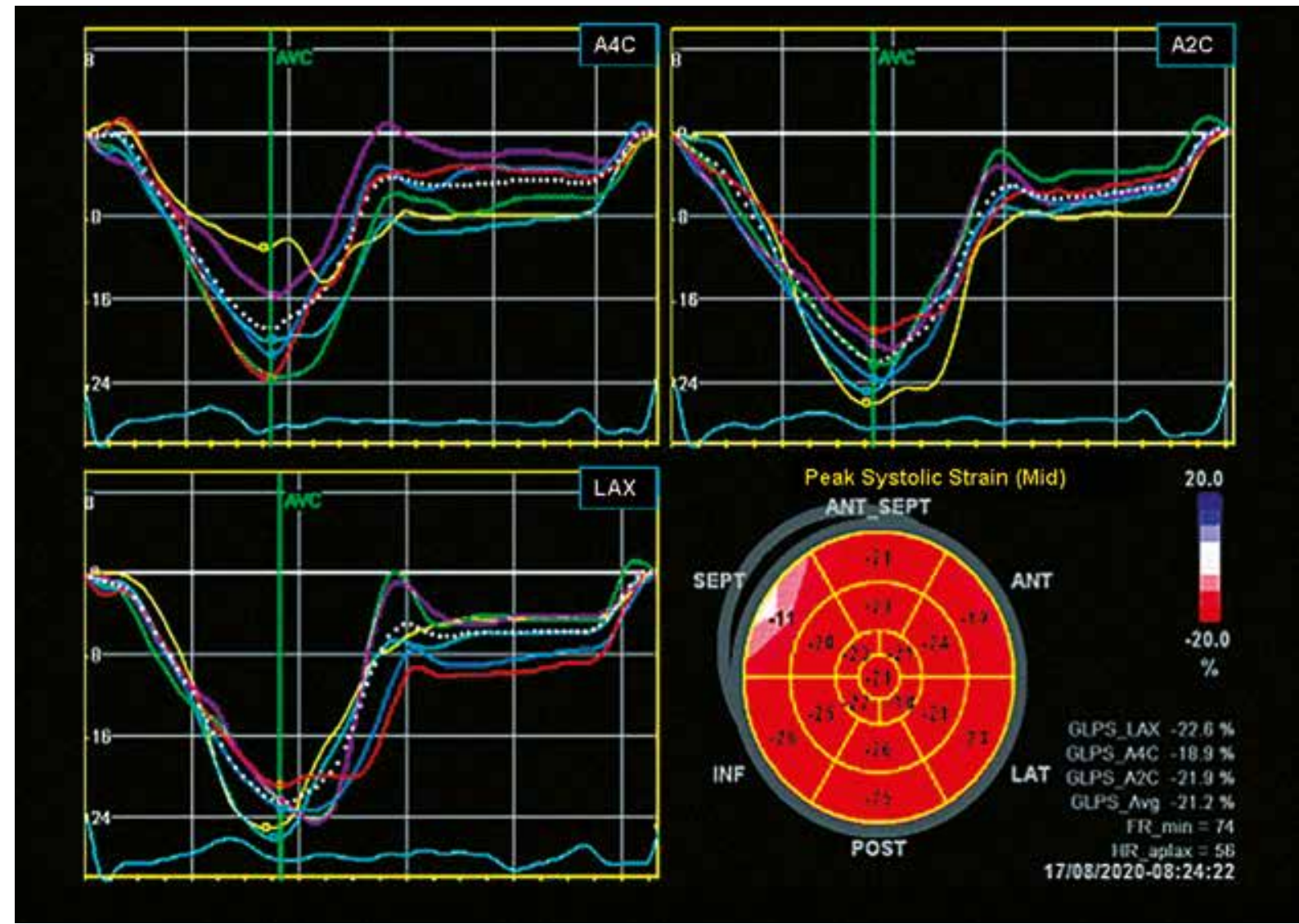

Fig. 1. Global longitudinal strain (GLS) polar map image of a cirrhotic patient with preserved systolic function and normal GLS (GLS average 21.2\%) 
to measure early (E) and late (A) diastolic filling velocities, their ratio (E/A ratio), and the early wave deceleration time (DT) [12].

The tissue Doppler imaging (TDI) program was set to pulse-wave Doppler mode. Filters were set to exclude high frequency signals. Gains were minimized to allow a clear tissue signal with minimal background noise. The TDI of the diastolic velocities was obtained from the apical 4-chamber view positioning the recorded wall in the centre of the sector and placing a $1.5-\mathrm{mm}$ sample volume at the septal corner of the mitral valve annulus. The angle between the Doppler beam and the longitudinal motion of the septal mitral valve annulus was minimized, as well. All Doppler parameters were recorded at a horizontal speed of $100 \mathrm{~mm} / \mathrm{s}$. The average values obtained for at least three consecutive cardiac cycles were taken into consideration. Early diastolic peak velocity of septal mitral annulus (septal $\mathrm{E}^{\prime}$ ) was obtained and the E/e' ratio was derived [12]. Analysis of strain rate (SR) parameters was performed offline by the same operator (M.C.). Placing the region of interest on the medial corner of the mitral annulus we assessed its peak systolic tissue velocity (septal $\mathrm{S}^{\prime}$ ). Placing the region of interest $(6 \mathrm{~mm} \times 4 \mathrm{~mm})$ on the basal portion of the inferior interventricular septum we assessed septal peak systolic strain (septal strain) and the systolic strain rate (septal SRs).

The measurement of these echocardiographic parameters was shown to be highly reproducible in our laboratory, as already reported $[13,14]$.

\section{Definitions and calculation of parameters}

The following parameters were calculated: mean arterial pressure (MAP) (systolic pressure $+2 \times$ diastolic pressure/3); stroke volume (SV) was computed as the difference between end-diastolic and end-systolic LV volume and used as a direct indicator of LV volume load. Cardiac output (CO) was calculated as the product of SV and heart rate; cardiac index $(\mathrm{CI})$ was calculated as $\mathrm{CO}$ adjusted by the BSA; systemic vascular resistance index (SVRI) was calculated as the product of MAP and 80/CI [4]; stroke work (SW), a measure of total cardiac workload, was calculated as the product of systolic blood pressure (pressure load) and SV (volume load) and converted into gram-metres per beat by multiplying by the conversion factor 0.0014 [14].

Myocardial mechano-energetic efficiency (MEE) was estimated as follows: SV ( $\mathrm{z}$-derived)/(heart rate $x$ $0.6)$ and divided by LVM to obtain an estimate of MEE per gram of LVM $(\mathrm{MEEi})(\mathrm{ml} / \mathrm{s} \times \mathrm{g})$ [15].

Advanced diastolic dysfunction was diagnosed if $\geq 3$ of the following criteria were met: left atrial vol- ume index $>34 \mathrm{ml} / \mathrm{m}^{2}$, tricuspid regurgitation velocity $>2.8 \mathrm{~m} / \mathrm{s}$, septal $\mathrm{e}^{\prime}$ velocity (early diastolic myocardial velocity on TDI) $<7 \mathrm{~cm} / \mathrm{s}, \mathrm{E} / \mathrm{e}^{\prime}$ (early diastolic transmitral and myocardial velocity on TDI ratio ) $>15$ [7].

\section{Statistical analysis}

The data are expressed as mean \pm SD (or SEM, or median and range), as appropriate. All echocardiographic and haemodynamic parameters showed a normal distribution, which was formally verified by Kolmogorov-Smirnov test. Student's $t$-test or Wilcoxon rank sum test was applied to compare quantitative variables, and $\chi^{2}$ analysis for categorical variables. Significance was set at $p<0.05$. The statistical analysis was performed using SPSS 18 for Windows (SPSS Italy Inc., Bologna, Italy).

\section{Results}

Between 2009 to 2012 we recruited 162 consecutive outpatients with cirrhosis. As shown in Figure 2, patients were excluded if they had arterial hypertension $(n=11)$, history of cardiovascular disease $(n=5)$, diabetes mellitus $(n=16)$, heart valve disease $(n=7)$, or coronary heart disease $(n=5)$. Three patients were lost to follow-up. Of the remaining 115 patients only in 83 were good and standardized 2D echocardiographic images available to permit correct speckle tracking analysis according to the EACVI/ASE guidelines [11].

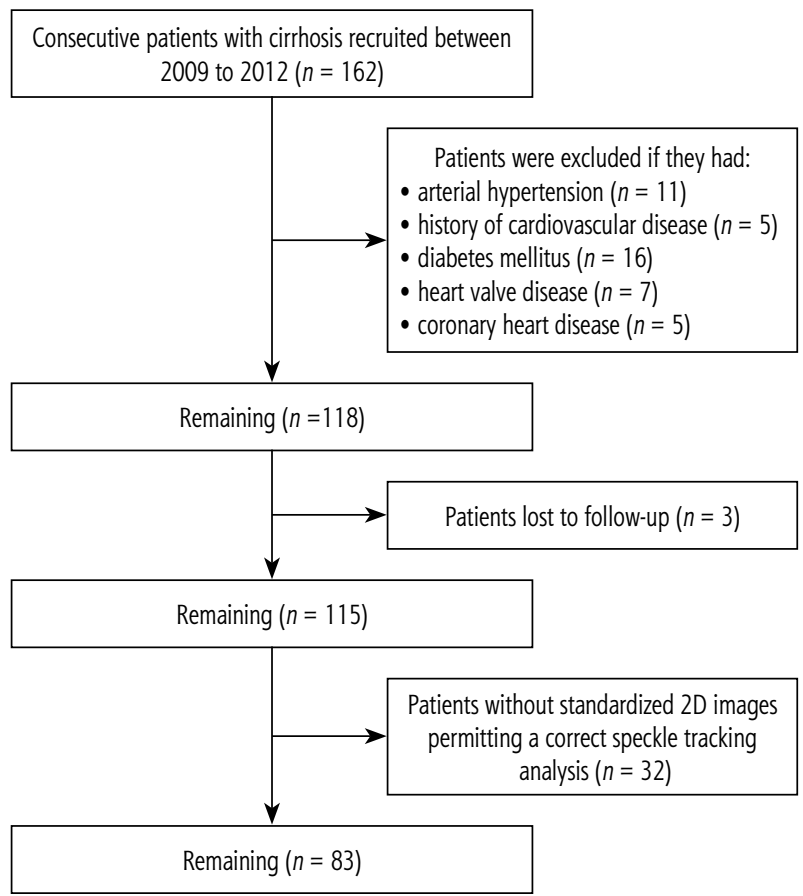

Fig. 2. Study population 
These 83 patients were mostly males, in different stages of liver disease of different aetiology. As expected, cirrhotic patients showed a reduction of MAP and peripheral vascular resistance, and an increase of heart rate and QTc, LA dimension, LV dimension and mass, SV, SW, and CO as compared with healthy subjects (Tables 3 and 4).

\section{Diagnosis and prevalence of cirrhotic cardiomyopathy}

As regards the systolic dysfunction, no patient presented LVEF $\leq 50 \%$. A GLS $<18 \%$ or $>22 \%$ was evident in 21 patients (25\%) independently of aetiology of cirrhosis or the presence/absence of ascites. As re-

Table 3. Clinical, demographic and biochemical features of cirrhotic patients with and without cirrhotic cardiomyopathy (CCM)

\begin{tabular}{|c|c|c|c|c|}
\hline Variable & CCM present $(n=24)$ & CCM absent $(n=59)$ & $P$ & Healthy subjects $(n=46)$ \\
\hline Age (years) & $60 \pm 12$ & $56 \pm 10$ & NS & $55 \pm 10$ \\
\hline Gender, male (\%) & 79 & 70 & NS & 48 \\
\hline Alcoholic aetiology (\%) & 50 & 43 & NS & 0 \\
\hline MELD & $12 \pm 7$ & $12 \pm 5$ & NS & 0 \\
\hline Presence of ascites (\%) & 25 & 14 & NS & 0 \\
\hline Dead after 6 years FW (\%) & 38 & 36 & NS & 0 \\
\hline Development of HRS (no. of patients and \% in the group) & $1(4 \%)$ & $4(7 \%)$ & NS & 0 \\
\hline $\mathrm{BMI}\left(\mathrm{kg} / \mathrm{m}^{2}\right)$ & $26 \pm 3$ & $26 \pm 4$ & NS & $25 \pm 5$ \\
\hline QTC & $452 \pm 32$ & $453 \pm 27$ & NS & $410 \pm 21$ \\
\hline $\mathrm{MAP}(\mathrm{mmHg})$ & $93 \pm 11$ & $96 \pm 13$ & NS & $97 \pm 7$ \\
\hline Heart rate (bpm) & $71 \pm 11$ & $69 \pm 10$ & NS & $67 \pm 10$ \\
\hline Creatinine $(\mu \mathrm{mol} / \mathrm{l})$ & $89 \pm 37$ & $96 \pm 80$ & NS & NA \\
\hline Aldosterone (ng/dl) & $59(32-86)$ & $66(48-84)$ & NS & NA \\
\hline
\end{tabular}

The data are reported as mean $\pm S D$ or median (and interquartile range) as appropriate. HRS - hepatorenal syndrome, MAP - mean arterial pressure, NA - not available

Table 4. Haemodynamic and echocardiographic features of cirrhotic patients with and without cirrhotic cardiomyopathy (CCM)

\begin{tabular}{|c|c|c|c|c|}
\hline Variable & CCM present $(n=24)$ & CCM absent $(n=59)$ & $P$ & Healthy subjects ( $n=46$ ) \\
\hline MWFS & $16 \pm 2$ & $17 \pm 2$ & NS & $17 \pm 1$ \\
\hline MEE (ml/s) & $1.80 \pm 0.46$ & $1.91 \pm 0.51$ & NS & $1.78 \pm 0.49$ \\
\hline MEEi $(\mathrm{ml} / \mathrm{s} \times \mathrm{g})$ & $0.90 \pm 0.22$ & $0.99 \pm 0.27$ & NS & $1.02 \pm 0.17$ \\
\hline LA volume $\left(\mathrm{ml} / \mathrm{m}^{2}\right)$ & $34 \pm 3$ & $35 \pm 4$ & NS & $29 \pm 4$ \\
\hline LVEDVol (ml) & $108 \pm 19$ & $109 \pm 20$ & NS & $96 \pm 16$ \\
\hline LVM/height ( $\left(\mathrm{g} / \mathrm{m}^{2.7}\right)$ & $47 \pm 10$ & $47 \pm 10$ & NS & $42 \pm 7$ \\
\hline SV (ml/beat) & $75 \pm 12$ & $77 \pm 14$ & NS & $68 \pm 11$ \\
\hline SW (g/beat) & $137 \pm 24$ & $143 \pm 36$ & NS & $126 \pm 24$ \\
\hline CO (1/min) & $5.28 \pm 1.03$ & $5.35 \pm 1.20$ & NS & $4.60 \pm 0.85$ \\
\hline GLS & $21 \pm 3$ & $20 \pm 1$ & NS & $20 \pm 1$ \\
\hline $\mathrm{DT} / \mathrm{HR}$ & $3.6 \pm 1.1$ & $3.7 \pm 1.0$ & NS & $3.6 \pm 1.0$ \\
\hline$E / e^{\prime}$ & $11 \pm 3$ & $10 \pm 3$ & NS & $8 \pm 2$ \\
\hline $\mathrm{E} / \mathrm{A}$ & $1.05 \pm 0.34$ & $1.16 \pm 0.11$ & NS & $1.28 \pm 0.31$ \\
\hline
\end{tabular}

The data are reported as mean $\pm S D$. CO - cardiac output, DT/HR - deceleration time of the $E$ wave/heart rate, GLS - global longitudinal strain, $E / A$ - early and late diastolic velocity ratio, LA - left atrium, LVEDVol - left ventricular end-diastolic volume, LVM - left ventricular mass, MEE - myocardial mechano-energetic efficiency indexed for LVM, MWFS - midwall fractional shortening, SV - stroke volume, SW - stroke work. 
gards the parameters used to define diastolic function, an increased LA volume $\left(>34 \mathrm{ml} / \mathrm{m}^{2}\right)$ was evident in 26 patients (31\%); tricuspid regurgitation velocity $>2.8 \mathrm{~m} / \mathrm{s}$ was evident in 10 patients (12\%), septal $\mathrm{e}^{\prime}$ velocity $<7 \mathrm{~cm} / \mathrm{s}$ was evident in 15 patients $(18 \%), \mathrm{E} / \mathrm{e}^{\prime}$ $>15$ was evident in 9 patients (11\%). Considering together the above parameters, advanced diastolic dysfunction was evident in 8 patients (10\%).

Overall, the prevalence of CCM defined according to all the above parameters was $29 \%$.

\section{Comparison of patients with and without cirrhotic cardiomyopathy}

Tables 3 and 4 present the main clinical, biochemical, haemodynamic and echocardiographic parameters of cirrhotic patients divided according to the presence or absence of CCM. No significant differences emerged between the two groups. In particular, no differences were found as regards cardiac dimensions, subclinical systolic dysfunction (MWFS and GLS), cardiac work (SV, SW and CO), and myocardial mechano-energetic efficiency (MEEi). Moreover, other accepted indexes of diastolic function (E/e', E/A, DT/HR) were found to be similar in patients with and without CCM.

\section{Survival according to the diagnosis of cirrhotic cardiomyopathy}

Among the 83 patients considered in this study, during a median follow-up of 6 years (range: 20 days7 years) 30 patients died (36\%) and 5 patients developed type 1 HRS. Main causes of death were complications of cirrhosis (gastrointestinal haemorrhages, hepatorenal syndrome, liver failure, sepsis, hepatocellular carcinoma). Patients with and without CCM presented similar incidence of death after 6 years of follow-up. Of the 5 patients who developed HRS only 1 presented CCM (Table 3). In a sub-analysis we considered only the 47 patients with cirrhosis and ascites who were on the list for liver transplantation. Of them, 17 presented CCM and 30 did not. The mortality rates in the two groups were $41 \%$ in patients with CCM and $43 \%$ in patients without CCM (NS). Paradoxically, the 17 patients on the transplant list with CCM presented a lower incidence of HRS as compared with patients without CCM (6\% vs. $13 \%$, respectively) during the follow-up.

\section{Discussion}

Cardiac complications are common after orthotopic liver transplantation (OLT), ranging from $7 \%$ to
$70 \%$, and lead to considerable mortality and morbidity [3].

Perioperative and postoperative heart failure with reduced left ventricular ejection fraction $(\mathrm{HFrEF})$ has been observed in $3 \%$ to $14 \%$ patients $[16,17]$ despite the majority of them presenting normal LV function before OLT, since patients with overt systolic dysfunction are often declined for OLT.

In a single-centre cohort of 176 consecutive patients who underwent OLT, post-OLT HFrEF (defined as $\mathrm{EF} \leq 40 \%$ with acute heart failure symptoms) was present in $14 \%$ with a median of 5 days and was associated with a 7-fold increase in 1-year mortality [17].

Cirrhotic cardiomyopathy is considered an important risk factor for early post-transplant $\operatorname{HFrEF}[17,18]$ since it may manifest with unexpected and often severe LV systolic and diastolic dysfunction, sometimes rapidly arising after transplant when the redistribution of significant blood volumes leads to marked right and LV volume overload, and a contemporary increase in afterload due to the rapid postoperative reversion of systemic vasodilation. Hence, the pre-LT diagnosis of CCM is fundamental to identify patients at higher risk of developing perioperative and postoperative HFrEF.

The newly proposed criteria to define CCM consider both subclinical systolic dysfunction and advanced diastolic dysfunction.

As regards the systolic dysfunction, since in our cohort of cirrhotic patients in different stages of liver disease none presented a $\mathrm{LVEF}<50 \%$ it is clear that a low LVEF is not a useful criterion to define CCM and should not be considered in the guidelines. At variance, altered GLS (as defined if $<18 \%$ or $>22 \%$ ) was evident in $25 \%$ independently of aetiology of cirrhosis or the presence/absence of ascites. However, after a 6-year follow-up the percentage of patients who died was similar in patients with and without altered GLS or advanced diastolic dysfunction at baseline (i.e. with and without CCM so defined), thus suggesting a poor relevance of this classification in terms of prognosis, at least in cirrhotic patients. It should be noted that the evidence of an altered GLS at rest in cirrhotic patients is limited and conflicting. Some studies showed normal longitudinal strain when compared with controls $[19,20]$, while others reporting a reduced longitudinal strain compared with controls but without a difference between compensated vs decompensated patients (i.e. between Child-Pugh A, B, and C grade cirrhosis) [2123] or an increased GLS at rest (i.e. higher LV contractility) in more advanced liver disease (Child-Pugh C) correlating with a worse prognosis $[24,25]$. In the newly proposed classification a GLS $<18 \%$ and $>22 \%$ has been considered diagnostic of CCM [7]. 
As regards the diastolic dysfunction, we previously reported that $\mathrm{DD}$, as defined according to both previous (ASE 2009) [12] and current guidelines (ASE/ EACVI 2016) [26], does not correlate with poor prognosis in a large cohort of cirrhotic patients followed for 6 years [14,27]. This is in line with the results of other groups $[23,28,29]$ which observed an independent predictive value of left atrial dimension [29] or E/e' [28] but not of the above definitions of DD proposed by current guidelines [26]. Moreover, the prevalence of DD according to the algorithms below demonstrated a wide variability independently of the severity of liver disease [30], probably because none of these algorithms was developed in patients with cirrhosis, but they have been validated in patients with symptomatic cardiovascular diseases, aiming at identifying increased cardiac filling pressures, a condition which is probably uncommon in asymptomatic cirrhotic patients with normal LVEF and with central hypovolemia.

\section{Conclusions}

In conclusion, we believe that the recently proposed new criteria to define CCM, predominantly based on altered GLS at rest, are mainly descriptive, with poor relevance in terms of prognosis and useless for the clinical management of cirrhotic patients, particularly in pre-transplant evaluation.

Moreover, this classification does not permit to clarify whether CCM represents a disease per se or a symptom of cirrhosis. If the aim is to identify a pathophysiological condition, i.e. subclinical cardiac dysfunction, which could affect the outcome during/after OLT, we believe that a stress test (such as a stress-echocardiography with low-dose dobutamine) assessing not the presence/absence of inducible ischaemia (i.e. coronary artery disease detection) but the cardiac contractile reserve could allow one to distinguish patients with a blunted cardiac response (i.e. with CCM) who could be at higher risk of new-onset HF. Hence, we suggest modifying the proposed new criteria by removing the assessment of LVEF and adding stress echocardiography assessing the cardiac contractile reserve. Further research in this field is required to better clarify which is the best stress test to assess the cardiac contractile reserve and the predictive value of a blunted cardiac response to stress for the development of perioperative and postoperative HFrEF.

\section{Limitations and strengths}

We recognize that a single-centre, retrospective design could limit the robustness of the findings.
Strengths of this study to be underscored include the long follow-up and the large sample size of phenotypically well-characterized cirrhotic patients; the use of state-of-the art echo-Doppler techniques entailing TDI and speckle tracking analysis; and the fact that all of the echocardiograms were performed by a single experienced cardiologist.

\section{Acknowledgments}

We acknowledge Dr Antonella Cecchetto M.D. (Cardiology, Department of Cardiology Thoracic and Vascular Sciences and Public Health, University of Padua, Italy) for her valuable contribution to the assessment of global longitudinal strain.

\section{Disclosure}

The authors declare no conflict of interest.

\section{References}

1. Moller S, Henriksen JH. Cirrhotic cardiomyopathy. J Hepatol 2010; 53: 179-190.

2. Krag A, Bendtsen F, Henriksen JH, et al. Low cardiac output predicts development of hepatorenal syndrome and survival in patients with cirrhosis and ascites. Gut 2010; 59: 105-110.

3. Feltracco P, Barbieri S, Carollo C, et al. Early circulatory complications in liver transplant patients. Transplant Rev 2019; 33: 219-230.

4. Torregrosa M, Aguade S, Dos L, et al. Cardiac alterations in cirrhosis: reversibility after liver transplantation. J Hepatol 2005; 42: 68-74.

5. Torp H, Echn DRT, Crosby J, et al. Noninvasive myocardial strain measurement by speckle tracking echocardiography and tagged magnetic resonance imaging. J Am Coll Cardiol 2006; 47: 789-793.

6. Biering-Sørensen T, Biering-Sørensen SR, Olsen FJ, et al. Global longitudinal strain by echocardiography predicts long-term risk of cardiovascular morbidity and mortality in a low risk general population: the Copenhagen city heart study. Circ Cardiovasc Imaging 2017; 10: e005521.

7. Izzy M, VanWagner LB, Lin G, Altieri M, et al. Redefining cirrhotic cardiomyopathy for the modern era. Hepatology 2020; 71: 334-345.

8. Salerno F, Gerbes A, Gines P, et al. Diagnosis, prevention and treatment of hepatorenal syndrome in cirrhosis. Gut 2007; 56: 1310-1318.

9. Lang RM, Bierig M, Devereux RB, et al. Recommendations for chamber quantification. Eur J Echocardiogr 2006; 7: 79-108.

10. De Marco M, Chinali M, Romano C, et al. Increased left ventricular mass in pre-liver transplantation cirrhotic patients. J Cardiovasc Med 2008; 9: 142-146

11. Voigt JU, Pedrizzetti G, Lysyansky P, et al. Definitions for a common standard for 2D speckle tracking echocardiography: consensus document of the EACVI/ASE/Industry Task Force to standardize deformation imaging. J Am Soc Echocardiogr 2015; 28: 183-193.

12. Nagueh SF, Appleton CP, Gillebert TC, et al. Recommendations for the evaluation of left ventricular diastolic function by echocardiography. Eur J Echocardiogr 2009; 10: 165-193. 
13. Cesari M, Letizia C, Angeli P, et al. Cardiac remodeling in patients with primary and secondary aldosteronism; a tissue doppler study. Circ Cardiovasc Imaging 2016; 9: e004815.

14. Cesari M, Frigo AC, Tonon M, et al. Cardiovascular predictors of death in patients with cirrhosis. Hepatology 2018; 68: 215-223.

15. de Simone G, Izzo R, Losi MA, et al. Depressed myocardial energetic efficiency is associated with increased cardiovascular risk in hypertensive left ventricular hypertrophy. J Hypertens 2016; 34: 1846-1853.

16. Ripoll C, Yotti R, Bermejo J, et al. The heart in liver transplantation. J Hepatol 2011; 54: 810-822.

17. Sakr AE, Fraser GE, Doctorian TP, et al. Predictors of systolic heart failure and mortality following orthotopic liver transplantation: a single-center cohort. Transplant Proc 2019; 51: 19501955.

18. Silvestre OM, Farias AQ, Bacal F. Early-onset and late-onset heart failure after liver transplantation. Liver Transplant 2014; 20: 122.

19. Rimbaş RC, Baldea SM, Guerra RDGA, et al. New definition criteria of myocardial dysfunction in patients with liver cirrhosis: a speckle tracking and tissue doppler imaging study. Ultrasound Med Biol 2018; 44: 562-574.

20. Pagourelias ED, Sotiriou P, Papadopoulos CE, et al. Left ventricular myocardial mechanics in cirrhosis : a speckle tracking echocardiographic study. Echocardiography 2016; 33: 223-232.

21. Chen Y, Chan AC, Chan S, et al. A detailed evaluation of cardiac function in cirrhotic patients and its alteration with or without liver transplantation. J Cardiol 2016; 67: 140-146.

22. Sampaio F, Pimenta J, Bettencourt N, et al. Systolic and diastolic dysfunction in cirrhosis: a tissue-Doppler and speckle tracking echocardiography study. Liver Int 2013; 33: 1158-1165.

23. Nazar A, Guevara M, Sitges M, et al. LEFT ventricular function assessed by echocardiography in cirrhosis: relationship to systemic hemodynamics and renal dysfunction. J Hepatol 2013; 58: 51-57.

24. Jansen C, Cox A, Schueler R, et al. Increased myocardial contractility identifies patients with decompensated cirrhosis requiring liver transplantation. Liver Transpl 2018; 24: 15-25.

25. Mechelinck M, Hartmann B, Hamada S, et al. Global longitudinal strain at rest as an independent predictor of mortality in liver transplant candidates: a retrospective clinical study. J Clin Med 2020; 9: 2616.

26. Nagueh SF, Smiseth OA, Appleton CP, et al. Recommendations for the evaluation of left ventricular diastolic function by echocardiography: an update from the American Society of Echocardiography and the European Association of Cardiovascular Imaging. J Am Soc Echocardiogr 2016; 29: 277-314.

27. Cesari M, Fasolato S, Rosi S, et al. Cardiac dysfunction in patients with cirrhosis : is the systolic component its main feature ? Eur J Gastroenterol Hepatol 2015; 27: 660-666.

28. Ruiz-del-Arbol L, Achecar L, Serradilla R, et al. Diastolic dysfunction is a predictor of poor outcomes in patients with cirrhosis, portal hypertension, and a normal creatinine. Hepatology 2013; 58: 1732-1741.

29. Merli M, Torromeo C, Giusto M, et al. Survival at 2 years among liver cirrhotic patients is influenced by left atrial volume and left ventricular mass. Liver Int 2017; 37: 700-706.

30. Cesari M. Reply to the letter of Rimbaş RC et al. Hepatology 2019; 69: 2715-2717. 